\title{
HOMOTOPY AND UNIFORM HOMOTOPY. II
}

\author{
ALLAN CALDER AND JERROLD SIEGEL
}

\begin{abstract}
An elementary proof of the Bounded Lifting Lemma is given, together with a proof that homotopy and uniform homotopy do not agree for maps into compact spaces with infinite fundamental groups even though they can agree for maps into a noncompact space with infinite fundamental group.
\end{abstract}

The purpose of this paper is two-fold: (1) To give a short, unified and a great deal more transparent proof of the main geometrical results of [1], upon which all of [1] and [2] depend. (2) To give a proof that if $Y$ is compact and $\pi_{1} Y$ is infinite then $[\beta-, Y]$ is not a homotopy functor. It follows that the result of [1] concerning the relation between homotopy and uniform homotopy for finite-dimensional normal spaces is best possible. We wish to thank J. Keesling for his observation with regard to (2).

A fibration $p: E \rightarrow B$, by which we will mean a (Hurewicz) fibration such that $B$ has a numerable covering $\left\{U_{\alpha}\right\}$ with $p^{-1}\left(U_{\alpha}\right)$ trivial in the sense of Dold [4], is said to have the bounded lifting property (BLP) with respect to a subcategory $\mathcal{T}$ of $\mathcal{T} \mathcal{P}$, the category of topological spaces and maps, if for every space $X$ in $\mathcal{T}$ and map $f: X \rightarrow E$ such that $p f$ is bounded there exist a bounded map $g: X \rightarrow E$ which is homotopic to $f$ over $p$. (A bounded map is one for which the closure of the image is compact.) That is to say that any lift to $E$ of a bounded map into $B$ is homotopic over $p$ to a bounded map. We say $p$ has $\operatorname{BLP}(\mathcal{T})$.

THeOREM 1 [1, (2.3) AND (3.3)]. Let $F$ be the fiber of $p: E \rightarrow B$; then (1) if $F$ has the homotopy type of a compact space then $p$ has BLP( $\mathcal{P} \odot)$, (2) if $F$ has the homotopy type of a $C W$-complex of finite type (i.e. finitely many cells in each dimension) then $p$ has $B L P(f d N o r m)$. Here fdNorm denotes the category of finite dimensional normal spaces.

A space $Y$ is said to have the relative compressibility property (RCP) with respect to $\mathcal{T}$ if for any space $X$ in $\mathcal{T}$, subspace $A$ of $X$ and map $f: X \rightarrow Y$ such that $\overline{f(A)}$ is compact, there exists a homotopy $H: X \times I \rightarrow Y$ such that $H_{0}=f$ and $\overline{H((X \times\{1\}) \cup(A \times I))}$ is compact. We say that $Y$ has $\operatorname{RCP}(\mathcal{T})$.

Clearly, a compact space has $\operatorname{RCP}(\mathcal{T} \odot \mathscr{P})$ and if $Z$ has $\operatorname{RCP}(\mathcal{T})$ and $Z$ dominates $Y$ (or in particular if $Z$ is homotopically equivalent to $Y$ ) then $Y$ has $\operatorname{RCP}(\mathfrak{T})$. So the theorem will be a consequence of the following two lemmas.

Received by the editors November 14, 1978.

AMS (MOS) subject classifications (1970). Primary 55E05, 55F05; Secondary 54D30.

Key words and phrases. Homotopy, fibrations, bounded map, bounded lifting property, relative compressibility property, Stone-Cech compactification. 0002-9939/80/0000-0081/\$01.75 
LEMMA 1. If $T$ is closed under closed subspaces and $F$ has $R C P(\mathcal{T})$ then $p$ has $B L P(\mathcal{T})$.

\section{LEMMA 2. A CW-complex of finite type has $R C P($ fdNorm).}

Proof of Lemma 1. Let $X$ be in $\mathcal{T}$ and $f: X \rightarrow E$ a map such that $h=p f$ is bounded. By restricting to $\overline{h(X)}$ if necessary we may assume that $B$ is compact. By our definition of fibration, there exists a finite open cover $\left\{U_{i}\right\}_{i=1}^{n}$ of $B$ such that $p^{-1}\left(\bar{U}_{i}\right)$ is fiber homotopy equivalent to $\bar{U}_{i} \times F$. Let $\phi_{i}$ be such a homotopy equivalence and $\psi_{i}$ its inverse.

Let $\left\{V_{i}\right\}$ be an open covering of $B$ such that $\bar{V}_{i} \subset U_{i}$. Put $E_{i}=h^{-1}\left(\bar{U}_{i}\right)$ and $F_{i}=h^{-1}\left(\bar{V}_{i}\right)$. Further, let $G_{i}: p^{-1}\left(\bar{U}_{i}\right) \times I \rightarrow p^{-1}\left(\bar{U}_{i}\right)$ be a fiber homotopy from the identity to $\psi_{i} \phi_{i}$ and $\eta_{i}: B \rightarrow I$ be a map such that $\eta_{i}\left(B-U_{i}\right)=\{0\}$ and $\eta_{i}\left(\bar{V}_{i}\right)=$ $\{1\}$.

Suppose that we have defined $g_{i-1}: X \rightarrow E$ such that $g_{i-1}$ is homotopic to $f$ over $p$ and $\overline{g_{i-1}\left(\cup_{j<i} F_{j}\right)}$ is compact. Let $A=E_{i} \cap \cup_{j<i} F_{j}$ and let $H_{i}: E_{i} \times I \rightarrow \bar{U}_{i}$ $\times F$ be a fiber homotopy such that $H_{i}(x, 0)=\phi_{i} g_{i-1}$ and $\overline{H_{i}\left(\left(E_{i} \times\{1\}\right) \cup(A \times I)\right)}$ is compact. Such $H_{i}$ exist since $F$ has $\operatorname{RCP}(\mathcal{T})$ and $\bar{U}_{i}$ is compact.

Define $g_{i}: X \rightarrow E$ by

$$
g_{i}(x)= \begin{cases}G_{i}\left(g_{i-1}(x), 2 \eta_{i} h(x)\right), & \eta_{i} h(x) \in\left[0, \frac{1}{2}\right] \\ \psi_{i} H_{i}\left(x, 2 \eta_{i} h(x)-1\right), & \eta_{i} h(x) \in\left[\frac{1}{2}, 1\right]\end{cases}
$$

Then $g_{i}$ is homotopic to $g_{i-1}$ (and hence to $f$ ) over $p$ and $\overline{g_{i}\left(\cup_{j<i} F_{j}\right)}$ is compact as it is contained in $\overline{g_{i-1}\left(\cup_{j<i} F_{j}\right)} \cup \overline{\psi_{i} H_{i}((X \times\{1\}) \cup(A \times I))}$. Putting $g_{0}=f$, the result follows by induction up to $n$.

Proof of Lemma 2. Let $Y$ be a $C W$-complex of finite type and let $\phi: Y \rightleftarrows K: \psi$ be a homotopy equivalence and its inverse, where $K$ is a locally finite simplicial complex.

Suppose that $X$ is a finite-dimensional normal space, $A$ a subspace of $X$ and $f$ : $X \rightarrow Y$ a map such that $\overline{f(A)}$ is compact. Let $\mathcal{V}$ be the star cover of $K$ and $\mathcal{Q}$ a finite-dimensional cover of $X$ that refines $(\phi f)^{-1} \mathcal{V}$. Let $\pi: X \rightarrow \nu \mathscr{U}$ be a canonical projection of $X$ onto the nerve of $\mathscr{Q}$. Then there exists a simplicial map $\sigma$ : $\nu$ U $\rightarrow K$ such that $\sigma \pi$ is contiguous to $\phi f$.

Let $\Theta: X \times I \rightarrow K$ be the linear deformation (see [3, p. 354]), $\phi f$ to $\sigma \pi$ then $\Theta(A \times I) \cup \sigma \pi(X)$ is contained in some $m$-skeleton $K^{m}$ of $K$. Let $D: Y \times I \rightarrow Y$ be a homotopy from the identity to $\psi \phi$. Define $H: X \times I \rightarrow Y$ by

$$
H(x, t)= \begin{cases}D(f(x), 2 t), & t \in\left[0, \frac{1}{2}\right] \\ \psi \Theta(x, 2 t-1), & t \in\left[\frac{1}{2}, 1\right] .\end{cases}
$$

We may assume that $\psi$ is cellular so that $\psi\left(K^{m}\right) \subset Y^{m}$, the $m$-skeleton of $Y$, which is compact. Hence $H((X \times\{1\}) \cup(A \times I))$ is contained in a compact subset of $Y$, namely $Y^{m} \cup D(\overline{f(A)} \times I)$. 
REMARKs. 1. A slight modification of the proof of Lemma 2 shows that for any given $X$ one only needs that $F$ has a compact $(\operatorname{dim} X)$-skeleton.

2. The nature of the proof of Lemma 2 seems to indicate that Theorem 1 contains all the useful geometric information about the relation between homotopy and uniform homotopy in that it shows that it is very unlikely that there are other useful categories $\mathcal{S}$ and $\mathcal{T}$ such that all the spaces in $\mathcal{S}$ have $\operatorname{RCP}(\mathcal{T})$.

3. Part 2 of Theorem 1 is slightly stronger than (3.3) of [1] in that we do not require that $B$ has the homotopy type of a $C W$-complex.

As usual $\beta$ will denote the Stone-Čech compactification functor on the category of completely regular Hausdorff spaces.

THEOREM 2. If $Y$ is compact and $\pi_{1} Y$ is infinite then there is a homotopically nontrivial map from $\beta \mathbf{R}$ to $Y$. Hence $[\beta-, Y]$ is not a homotopy functor on any category that contains the real line $\mathbf{R}$.

Proof. Let $P Y$ denote the space of paths in $Y$ starting at $* \in Y$ and $p: P Y \rightarrow Y$ the map $p(\lambda)=\lambda(1)$. Then $p$ is a fibration with fiber $\Omega Y$, the space of loops at $*$. That a map $\beta f: \beta \mathbf{R} \rightarrow Y$ is homotopically trivial is equivalent to being able to factor it though $p$. This in turn is equivalent to being able to factor $f: \mathbf{R} \rightarrow Y$ through $p$ via a bounded map into $P Y,[1]$.

Since $\pi_{1} Y$ is infinite, $\Omega Y$ has infinitely many path components. Let $\left\{\sigma_{i}\right\}_{i=0}^{\infty} \subset$ $\Omega Y$ be such that $\sigma_{0}$ is the constant loop to $*$ and $\sigma_{i}$ and $\sigma_{j}$ are in distinct path components for $i \neq j$. Define $f: \mathbf{R} \rightarrow Y$ by $f(x)=\sigma_{i} \sigma_{i-1}^{-1}(x-i), x \in[i, i+1]$ and $f(x)=*, x \leqslant 1$. Since $Y$ is compact $f$ extends to $\beta \mathbf{R}$.

Now any lift $\phi$ of $f$ to $P Y$ must be unbounded as $\phi(i)$ and $\phi(j)$ must be in distinct path components of $\Omega Y$.

RemarK 4. The condition that $Y$ is compact is essential in Theorem 2 , since by [2, Theorem 3.4] for torsion abelian groups $G,[\beta-, K(G, 1)]$ is a homotopy functor on completely regular Hausdorff spaces, where $K(G, 1)$ is an EilenbergMac Lane space of type $(G, 1)$. In particular one could take $G=\mathbf{Q} / \mathbf{Z}$.

\section{REFERENCES}

1. A. Calder and J. Siegel, Homotopy and uniform homotopy, Trans. Amer. Math. Soc. 235 (1978), 245-269.

2. Kan extensions of homotopy functors, J. Pure Appl. Algebra 12 (1978), 253-269.

3. A. Dold, Lectures in algebraic topology, Springer-Verlag, Berlin and New York, 1972.

4. __ Partitions of unity in the theory of fibrations, Ann. of Math. (2) 78 (1963), 223-255.

Department of Mathematics, Birkbeck College, London WCl, England

Department of Mathematics, University of Missouri, St. Louis, Missouri 63121 (Current address of Jerrold Siegel)

Current address (Allan Calder): Department of Mathematics, New Mexico State University, Las Cruces, New Mexico 88003 